\title{
PLASMA MEASURES OF B-ENDORPHIN-LIKE IMMUNOREACTIVITY IN DEPRESSIVES AND OTHER PSYCHIATRIC SUBJECTS
}

\author{
J. Matthews, H. Akil, J. Greden, and S. Watson \\ Mental Health Research Institute \\ University of Michigan \\ Ann Arbor, Michigan 48109
}

(Received in final form June 14, 1982)

\section{Summary}

We report the utilization of a very sensitive radioimmunoassay for plasma B-END, which has enabled us to examine the suppressibility of B-END-like material, under dexamethasone challenge, on a population of depressed patients. We have found that B-END-like material in plasma is suppressed by dexamethasone in a psychiatric control population, whereas suppression is less likely in endogenously depressed patients. There also appears to be a dissociation between cortisol and B-END measures (tested at 4:00 PM) in the endogenously depressed group.

Several lines of evidence demonstrate an abnormality in the hypothalmic-pituitary-adrenal axis in patients with endogenous depression $(1,2,3)$. There are multiple levels where the defect might be localized. The dexamethasone suppression test (DST) measures the pituitary-adrenal interface. This paper attempts to move the study of the endocrinology of depression to the level of the pituitary and brain. Thus, we are interested in measuring beta-endorphin since it is part of the same precursor molecule, pro-opiomelanocortin, as ACTH $(4,5)$. We have used a dexamethasone challenge to beg in to look at the regulation of the pituitary pro-opiomelanocortin system. Although one study reports no suppression with dexamethasone in normals, the plasma radioimmunoassay is difficult and may lead to false negatives (6). We have therefore addressed here the following two questions: a) Does dexamethasone suppress B-END in human plasma? and b) Is the pattern of B-END suppression altered in endogenous depression?

\section{Methods}

Blood collection was carried out via angiocatheter into chilled EDTA containing vacutainers. The samples were centrifuged at $4 \mathrm{C}$ for 10 minutes and were then aliquoted into plastic storage vials and acidified to a pHof 2.0 with $1 \mathrm{~N} \mathrm{HCl}$. The acidified serum was stored at $-70^{\circ} \mathrm{C}$ until extraction. We used Sep Pak $C_{1}$ cartridges (Waters) for qur extraction procedure which provided 908 recovery with $\mathrm{H}^{3}-\mathrm{B}-\mathrm{END}$ spiked human serum (9). 
The radioimmunoassay was carried out in $150 \mathrm{mM}$ Na phosphate buffer with 18 human serum albumin at $\mathrm{pH} 8.2$ under disequilibrium incubation. The antiserum (rabbit Brenda) was used at a final concentration of $1: 40,000$ and separation was carried out by immunoprecipitation with goat antirabbit IgG. Crossreactivity studies with our antibody showed 1008 crossreactivity with $\mathrm{B}-\mathrm{LPH}$ and $\mathrm{N}$-acetyl-B-END, 508 with B-END, and 308 with $\mathrm{N}$-acety1-B-END $27^{\prime}$ whereas there is no cros ' reactivity with alpha-END, ${ }^{2}$ gamma-END, B-END ${ }^{\prime}$, B-END $_{2} 7^{\prime}$ methionine enkephalin, or alpha-melanocyte-stimulating hormoné. The assay is capable of measuring < 1.5 fmoles/assay tube (normal resting levels of B-END-like immunoreactivity are 2-5 fmoles $(\mathrm{mI})$. The validation of the assay and the chromatography of the material being measured have been described (9).

Using this procedure we studied a mixed psychiatric control population $(N=5)$ versus a group of endogenous depressives $(N=6)$. Under dexamethasone challenge serum cortisol and serum B-END levels were measured. An 18 gauge angiocatheter was inserted into an antecubital arm vein at 1:30 PM; $20 \mathrm{ml}$ blood samples were obtained at 20 minute intervals between $3: 30$ and $4: 30 \mathrm{PM}$. All subjects were then given $1 \mathrm{mg}$ of dexamethasone at 11:30 PM that evening and the catheter study was carried out in an identical fashion the following day. Both cortisol and B-END measures are reported as the means of the four time points between 3:30 and 4:30 PM. Thus: 1) simultaneous cortisol and B-END measures were obtained; 2) subjects were their own controls; and 3) repeated measures enable us to control for stress and pulsatile release of B-END. The 4:00 PM time period was chosen since this is the time when the dexamethasone suppression test is most sensitive in separating endogenously depressed patients from normal individuals.

\section{Results}

Table 1 shows the results. The non-endogenous psychiatric control group showed a mean suppression of their B-END levels of $458 \pm 8$ as compared to $188 \pm 8$ suppression for the endogenous deprēssives. The mean Hamilton rating for depression for the control group was $6 \pm 2$ and $24 \pm 1$ for the endogenous depressives. Further, there is an absence of overlap between pre- and post-dexamethasone B-END levels in the controls, whereas there was considerable overlap in endogenous depressives. The post-dexamethasone B-END values were very closely clustered in the controls in contrast to much greater variability in the endogenous depressives.

Since endogenously depressed patients usually exhibit cortisal values of greater than $5.0 \mathrm{ug} / \mathrm{dl}$ after dexamethasone, we have reviewed the B-END data for a similar cutoff. A conservative preliminary post-dexamethasone value of $2.5 \mathrm{fm} / \mathrm{ml}$ was above al1 non-endogenous post-dexamethasone values.

As summarized by Table 2, B-END levels above $2.5 \mathrm{fm} / \mathrm{ml}$ identified five out of the six endogenous depressives, whereas post-dexamethasone cortisol levels identified only two of the six endogenous depressives. Note that there is good agreement for the absence of abnormal B-END and cortisol responses in the non-endogenous groups, whereas the agreement for abnormal 
Table 1

Non-endogenous Psychiatric Controls

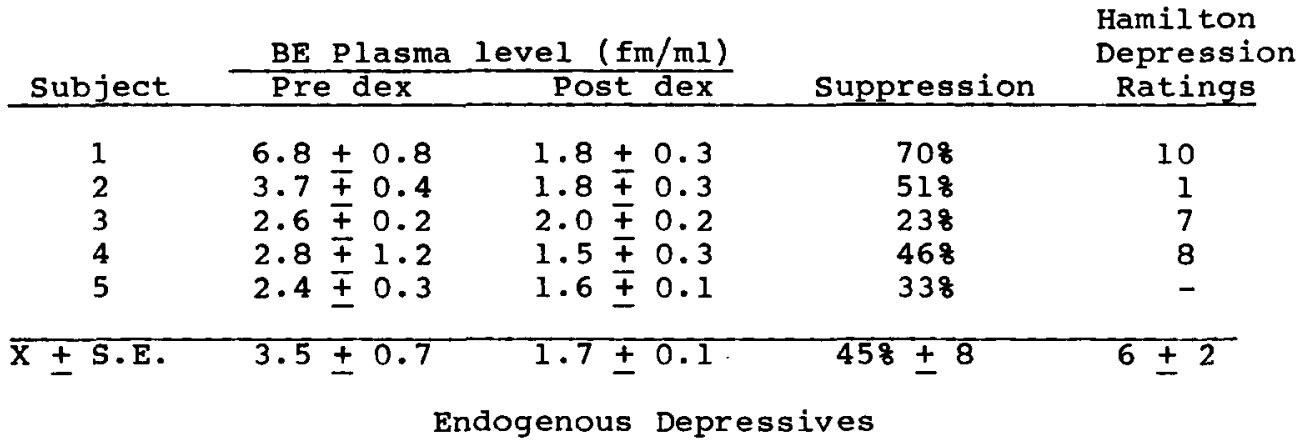

\begin{tabular}{cccc} 
Subject & $\frac{\text { B BE Plasma level }(\mathrm{fm} / \mathrm{ml})}{\text { Pre dex }} \quad$ Post dex $\quad$ Suppression $\begin{array}{c}\text { Hamilton } \\
\text { Depression } \\
\text { Ratings }\end{array}$ \\
\hline
\end{tabular}

\begin{tabular}{rrrrr}
6 & $3.6 \pm 0.4$ & $5.2 \pm 0.8$ & 08 & 21 \\
7 & $3.3 \pm 0.8$ & $3.2 \pm 1.7$ & 48 & 22 \\
8 & $10.2 \pm 1.6$ & $5.8 \pm 1.8$ & 438 & 38 \\
9 & $1.8 \pm 0.3$ & $1.2 \pm 0.2$ & 328 & 21 \\
10 & $5.4 \pm 0.9$ & $3.8 \pm 0.5$ & 318 & 18 \\
11 & $3.9 \pm 1.2$ & $4.0 \pm 0.9$ & 08 & 24 \\
\hline X S.E. & $4.7 \pm 1.3$ & $3.8 \pm 0.7$ & $188 \pm 8$ & $24 \pm 1$
\end{tabular}

Table 2

Abnormal BE

Suppression

$>2.5 \mathrm{fm} / \mathrm{ml}$

$\begin{array}{llll} & \# 1 & (-) & \\ \text { Non- } & \# 2 & (-) & \\ \text { endogenous } & \# 3 & (-) & 0 \\ & \# 4 & (-) & 5 \\ & \# 5 & (-) & \\ & \# 6 & (+) & \\ & \# 7 & (+) & \\ \text { Endogenous } & \# 8 & (+) & 5 \\ & \# 9 & (-) & 6 \\ & \# 10 & (+) & \\ & \# 11 & (+) & \end{array}$

Abnormal Cortisol Suppression $>5.0 \mathrm{ug} / \mathrm{dl}$

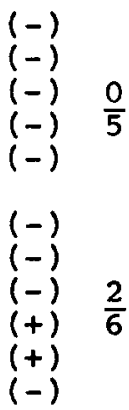

responses is poor in the endogenously depressed group. It should be noted, however, that the combination of B-END and cortisol measures around the 4:00 PM time point identifies all of the endogenously depressed patients. 


\section{Discussion}

These results suggest that B-END-like material in plasma is suppressed by dexamethasone in a non-endogenous psychiatric population, whereas suppression is less likely in endogenously depressed patients. Of interest is the observation that the combination of both cortisol and B-END-like measures identifies all of the endogenously depressed patients. The dissociation between cortisol and B-END-like values in the endogenously depressed patients warrants further investigation in view of reports suggesting that B-END and ACTH are secreted in equimolar amounts (7). Simultaneous measures of ACTH, B-END, and cortisol may help explain this lack of agreement and possibly help localize the defect. This discrepancy may also be related to the 3:30 to 4:30 PM sampling time since B-END and cortisol are regulated differently and have different half lives ( 8 ). There may be a time period at which cortisol and B-END measures are more tightly linked. Thus, the best time point post-dexamethasone B-END measurement needs to be determined. Twenty-four hour circadian rhythm studies are thus imperative. The large differences in the Hamilton ratings in the psychiatric controls versus the endogenous depressives suggests that severity of illnes may account for the lack of suppression in the endogenous depressives; thus, future studies need to control for this variable. Studies on normal controls and sex differences are now being carried out.

\section{Acknowledgements}

This work was supported by NIMH Grant \#R01-MH36168.

\section{References}

1. J.L. GIBBONS, Arch. Gen. Psychiatry 10 572-575 (1964)

2. B.J. CARROLL and B.M. DAVIES, Brit. Med. J. 1 789-791 (1970)

3. B.J. CARROLL, G.C. CURTIS and J. MENDELS, Arch. Gen. Psych. 33 1051-1058 (1976)

4. $\bar{B}$. EIPPER and R. MAINS, J. Supremolecular structure $\underline{8}$ 247-262 (1978)

5. S. NAKANISHI, A. INOUE, T. KITA, M. NAKAMURA, A.C.Y. CHANG, S.N. COHEN and S. NUMA, Nature 278 423-427 (1979)

6. N.H. KAHIN, S.C. RISCH, R.M. COHEN, T. INSEL and D.L. MURPHY, Science 209 827-828 (1980)

7. R. GUILLEMIN, T. VARGO, J. ROSSIER, S. MINICK, N. LING, C. RIVIER, W. VALE and F. BLOOM, Science 197 1357-1360 (1977)

8. M. ENDO, J. ENDO, M. NISHIKUBO, T. YAMAGUCHI and N. HATONANI, Psychoneuroendocrinology Workshop Conf. Int. Soc. Psychoneuroendocrinology, Mieken (Karger, Basel), 22-31 (1974)

9. C. CAHILL, J. MATTHEWS and H. AKIL, (submitted) 